\title{
Biomarkers in immunonutrition programme, is there still a need for new ones? A brief review
}

\author{
Patrice Forget $^{{ }_{1}}$, Garazi Echeverria ${ }^{* 1}$, Simone Giglioli ${ }^{* 1}$, Brigitte Bertrand ${ }^{2}$, Stephane Nikis ${ }^{3}$, Jean-Paul Lechat ${ }^{3}$ and Marc De Kock ${ }^{1}$ \\ 'Departments of Anesthesiology, Cliniques Universitaires Saint-Luc, Institute of Neuroscience av. Hippocrate 10-1821, Brussels 1200, Belgium \\ ${ }^{2}$ Department of Dietetics, Cliniques Universitaires Saint-Luc, Université Catholique de Louvain, Brussels 1200, Belgium \\ ${ }^{3}$ Grand Hopital de Charleroi, Grand'Rue 3, Charleroi 6000, Belgium \\ "Equal contributors
}

Correspondence to: Patrice Forget. Email: forgetpatrice@yahoo.fr

\section{Abstract}

Background and aims: Pre-existing malnutrition is one the most important factors affecting postsurgical complications, especially in cancer patients. The consequences of this on the immune function as well as on outcome could be reversed by immunonutrition. To help the clinician as a researcher, a routinely available biomarker (derived from clinical or biological data) would be of great importance.

Methods: We reviewed the potential markers that may routinely be used in perioperative immunonutrition programmes. A comprehensive approach was used to identify and discuss the potential markers, focusing on body mass and serum biomarkers.

Results: Body mass (including weight loss and body mass index) are predictive of complications, but not specifically to malnutrition. Serum markers, such as albumin, transthyretin, white blood cells counts, and C-reactive protein are not more specific. Composite scores, including the Nutritional Risk Index (NRI), the Prognostic Inflammatory and Nutritional Index (PINI), the modified Glasgow Prognostic Score (mGPS), the neutrophil-to-lymphocyte ratio (NLR), CD4 and CD8 lymphocytes counts, the platelet-to-lymphocyte ratio (PLR), the Prognostic Index $(\mathrm{PI})$, and the Prognostic Nutritional Index (PNI) are prognostic factors of outcome, but are not always correlated to immunonutrition effect.

Conclusions: In conclusion, there remains a lack of efficient and widely available monitoring of the effects of immunonutrition. To predict and monitor the effect of immunonutrition on immunity, efforts should be directed to the validation of routinely available tools to aid the implementation of advanced immune monitoring (like lymphocytes subpopulations counts) in clinical practices.

Keywords: immunonutrition, inflammation, biomarkers

ecancer 2015, 9:546 DOI: 10.3332/ecancer.2015.546

Copyright: $\odot$ the authors; licensee ecancermedicalscience. This is an Open Access article distributed under the terms of the Creative Commons Attribution License (http://creativecommons.org/licenses/by/3.0), which permits unrestricted use, distribution, and reproduction in any medium, provided the original work is properly cited. 


\section{Introduction}

First described in 1939, in patients undergoing major surgery (especially cancer patients and also all those undergoing intraperitoneal, intrathoracic prosthetic surgeries, which are increasingly proposed to older patients), pre-existing malnutrition is one of the most important factors affecting outcome [1]. Malnutrition decreases both cell-mediated and humoral immune response, increases the risk of infections, and affects wound healing, but can be reversed by re-nutrition [1, 2, 3, 4]. This is the basis of the concept of immunonutrition, with nutrients influencing immune cell functions and inflammatory pathways $[5,6]$. These immunoactive nutrients are $\omega$ - 3 polyunsaturated long-chain fatty acids, arginine, and antioxidants such as ascorbic acid [5]. Turnock et al showed that this type of immunonutrition in patients undergoing head and neck cancer surgery may decrease circulating pro-inflammatory cytokine concentrations, and increase postoperative anti-inflammatory mediators such as IL-10 [7].

A growing body of evidence supports the concept that an optimised nutritional support reduces the number of adverse outcomes following major cancer and non-cancer surgery [8, 9]. Importantly, the treatment effect varies depending on the patient population, the intervention, and the methodological quality of the study, including the frequent lack of an efficient biomarker able to monitor the effect of immunonutrition [10].

To improve outcome, a preoperative screening of the nutritional status and an early management of malnutrition are mandatory. Nevertheless, this screening remains difficult because there is absolutely no widely accepted criterion [11, 12].

To help the clinician as a researcher and to improve the use and the comprehension of the effects of immunonutrition, a biomarker would be of great importance. In this work, we review the potential markers that may be used to screen patients and to monitor the effect of immunonutrition in the perioperative period of major surgery.

\section{Potential biomarkers to detect malnutrition and to monitor the effects of immunonutrition}

The nutritional assessment is most often based on body mass, including weight loss and the calculation of body mass index (BMI), the albuminemia $[5,13,14,15,16,17]$. Here we show that, although many assessment tools exist, none is entirely satisfactory in terms of sensitivity and/or specificity.

\section{Body mass}

A weight loss greater than $10 \%$ of the usual weight or during the six last months is a predictive factor of postoperative complications in major surgery [13]. Even if it a widely used marker, one can say that severe malnutrition is sometimes present in the absence of weight loss, such as with ascites because of liver disease. The BMI is another important marker for the clinical evaluation of the nutritional status. A BMI lower than 18.5 is associated with an elevation of mortality and infection morbidity in cardiac and general surgery [14, 15], but again same flaws as for weight loss can be observed. Hence, a serum marker, such as albumin could be interesting.

\section{Serum protein levels: albumin and transthyretin (prealbumin)}

In the past, serum proteins and albumin level have been proposed as biological markers of malnutrition [5]. However, there are a lot of contradictions between the results of scientific studies and use of this marker in practice. For example, serum albumin level is not well correlated to protein mass, is affected by distribution and dilution (leading to discordance with other marker, especially after surgery), and is now more considered as a disease severity/prognostic marker as in cancer [31, 32]. Moreover, serum albumin does not always significantly increase after nutritional programmes [16]. Some authors proposed to replace it with transthyretin (prealbumin), more correlated to nutritional status [34]. Nevertheless, a role of transthyretin in the monitoring of the effect of immunonutrition was not confirmed [33].

To increase sensitivity, the combination of albumin levels with the patient body weight in the NRI was proposed [17]. 


\section{A combination: the NRI}

The NRI is a composite tool that allows a quantification of the risk of malnutrition. The NRI is calculated using the formula: NRI $=(1.489 \times$ serum albumin in $\mathrm{g} / \mathrm{L})+(41.7 \times$ current weight/usual weight $)$ [18]. It has been suggested that a NRI $>100$ indicates that the patient is not malnourished, while 97.5-100 indicates mild malnourishment, 83.5-97.5 indicates moderate malnourishment, and <83.5 indicates severe malnourishment [17]. The main advantage of this composite score is the potential correction of the masking effect on weight loss (i.e. absence of weight loss or even weight gain) because of oedemas in case of hypoalbuminemia. Nevertheless, this tool does not permit the monitoring of the potential therapeutic effect of nutrition, e.g. the reversing effect on immune function, whereas immunonutrition formulas have been developed to improve nutrition, cell immune function, and to modulate inflammation [10].

\section{White blood cells counts and C-reactive protein (CRP)}

White blood cell counts (WBCs) has been tested as possible parameters for the effects of immunonutrition in patients [8, 19, 20]. Unfortunately, no significant difference in WBC between the immunonutrition group and the normal nutrition group was showed by Giger et al during the first postoperative week [21].

On the other side, CRP as endotoxin levels (a potent trigger of acute phase reaction) were significantly lower in the treatment group on postoperative day 7 [21, 22]. In contrast, in a cohort of 116 patients undergoing major abdominal surgery (either receiving or not receiving immunonutrition), we were not able to confirm any difference in the pre- and postoperative CRP levels and WBCs [20].

\section{WBC and/or CRP are not definitive solutions}

The components of the systemic inflammatory response such as CRP, neutrophils, lymphocytes, and platelets, vary depending on the immune state of the patient. The Prognostic Inflammatory and Nutritional Index (PINI) combining orosomucoid, CRP, prealbumin, and albumin levels has been proposed as a solution [24, 25, 26]. Unfortunately, large and rapid changes in CRP in acute inflammation overestimate the values of PINI. Additionally, a decrease of orosomucoid is observed in hepatocellular insufficiency, acute kidney injury, with some medications (corticosteroids, NSAIDs, erythromycin, penicillin, $\alpha$-and beta-blockers, sulfasalazine) and in terminal stages of some neoplasias, which makes it an unreliable parameter [25]. As a result, the question remains about the interest of other inflammatory scores in this context $[27,28]$. This is an actual challenge in therapeutic and preventive cancer research to detect potential targets in chronic inflammatory disease which are essential links to promote inflammation-associated cancer [29].

\section{WBC-derived scores: from the neutrophil-to-lymphocyte ratio to the CD4:8 ratio}

The combinations of the components of the systemic inflammatory response to form prognostic scores based on inflammation such as the modified Glasgow Prognostic Score (mGPS), the neutrophil-to-lymphocyte ratio (NLR), the platelet-to-lymphocyte ratio (PLR), the Prognostic Index (PI), and the Prognostic Nutritional Index (PNI) have been evaluated and compared in cancer research [30, 31, 32]. It is known that systemic inflammation-based scores have prognostic value in a variety of cancers. In fact, an elevated mGPS, NLR, PLR, $\mathrm{PI}$, and PNI are independently predictive of a shorter cancer specific survival [31,32]. Recently, the interest of the NLR in the context of the possible influence of immunonutrition on inflammation was assessed [23]. As neutrophils and lymphocytes counts are related with the systemic inflammatory response, independently from the liver function but linked to malnutrition and zinc deficiency, it was logical to combine neutrophils and lymphocytes counts. A score like the NLR could potentially be more sensitive than WBCs counts [32]. However, clinical data did not confirm that NLR value is a good marker of the influence of immunonutrition on inflammation [23, 31]. 
To overcome this problem, some authors proposed to follow lymphocytes subpopulations. In head and neck cancer, changes suggesting that postoperative preservation of specific lymphocyte functions were associated with immunonutrition [33, 34]. This was especially observed for CD3 T cells and CD4 T helper cells counts, but also considering CD8 T suppressor cells counts and CD4:8 ratio [33, 34]. Nevertheless, the use of the lymphocytes subpopulations counts remains limited in routine practice.

\section{How to interpret these discrepancies?}

Immunonutrition is not always associated with a significant variation of the inflammatory markers, despite a presumed anti-inflammatory effect and influence on immune parameters. However, these studies have several limitations. First, looking at sensitive markers, like CRP, WBCs, or NLR in cancer patients, some of them are unavoidably outliers [20,23]. This phenomenon can be explained, for example, by emergent surgery, high inflammatory status (e.g. in sepsis). This fact can challenge any robust analysis. Second, heterogeneity is frequent, precluding definitive conclusion before analysis on large patients' cohorts. A typical high level of internal variability is seen in catabolic situations like after radiotherapy, chemotherapy (and tumour necrosis), immunosuppressive therapy, or changes in the distribution of leucocytes (like with the use of corticosteroids). All these limitations implicate the impracticability of the use of the parameters, described in this paper in a clinical setting. Nevertheless, we cannot definitively exclude their use in clinical research, as a condition for careful selection of patients' population.

\section{Conclusion}

In conclusion, there remains a lack of efficient and widely available monitoring of the effects of immunonutrition. To predict and monitor the effect of immunonutrition on immunity, efforts should be directed to the validation of routinely available tools for the implementation of advanced immune monitoring (like lymphocytes subpopulations counts) in clinical practice.

\section{Acknowledgments}

Nothing to declare.

\section{Statement of authorship}

PF, GE, SG, BB, MDK have participated to the design of the work, the selection of the sources, the analysis, the redaction, and approved the final version. SN and JPL participated in the reflexion, the critical appraisal of the sources, discussed the manuscript, and approved the final version.

\section{Conflicts of interest}

BG received from Nestlé SA covering for travel expenses to go to one congress. The other authors attest to no conflicts of interest.

\section{Financial support/Funding sources}

This work was exclusively supported by the Departments of Anesthesiology of the Cliniques Universitaires Saint-Luc, Brussels, Belgium. 


\section{References}

1. Correia MI and Waitzberg DL (2003) The impact of malnutrition on morbidity, mortality, length of stay hospital and costs evaluated trought a multivariate model analysis Clin Nutr 22(3) 235-9 DOI: 10.1016/S0261-5614(02)00215-7 PMID: 12765661

2. Mora RJF (1999) Malnutrition: organ and functional consequences Word J Surg 23(6) 530-5 DOI: 10.1007/PL00012343

3. Stundley HO (2001) Percentage of weight loss. A basic indicator of surgical risk in patients with chronic peptic ulcer Nutr Hosp 16(4) 141-3

4. Kamocki $Z$ et al (2013) The effect of perioperative immunonutrition on the phagocytic activity of blood platelets in advanced gastric cancer patients Clin Develop Immunol [Epub ahead of print] DOI: 10.1155/2013/435672

5. Marik PE and Zaloga GP (2010) Immunonutrition in high risk surgical patients: a systematic review and Analysis of the literature J Parent Ent Nutrition 34(4) 378-86 DOI: $10.1177 / 0148607110362692$

6. Bernard M and Aussel C (2007) Markers of malnutrition or markers of the complications related to the malnutrition? Nutrition Clinique et Metabolisme 21 52-9 DOI: 10.1016/j.nupar.2007.01.009

7. Doina K and Darwin D (2011) Specialized Nutrition Support Am Fam Physician 83(2) 173-83

8. Albers R and Bourdet-Sicard R (2013) Monitoring immune modulation by nutrition in the general population: identifying and substantiating effects on human health Br J Nutr 110 Suppl 2 S1-30 DOI: 10.1017/S0007114513001505 PMID: 23902657

9. Turnock A and Calder C (2013) Perioperative immunonutrition in well-nourished patients undergoing surgery for head and neck cancer: evaluation of inflammatory and immunologic outcomes Nutrients 5(4) 1186-99 DOI: 10.3390/nu5041186 PMID: 23571650 PMCID: $\underline{3705342}$

10. Heyland DK and Novak F (2001) Should immunonutrition become routine in critically ill patients? A systematic review of the evidence JAMA 286(8) 944-53 DOI: 10.1001/jama.286.8.944 PMID: 11509059

11. Weimann A (2006) ESPEN Guidelines on enteral nutrition. Surgery including organ transplantation Clin Nutr 25(2) 224-44 DOI: 10.1016/j.clnu.2006.01.015 PMID: 16698152

12. Waitzberg $D$ and Saito $H$ (2006) Postsurgical infections are reduced with specialized nutrition support World $J$ Surg $30(8)$ 1-13 DOI: $10.1007 / \mathrm{s} 00268-005-0657-\mathrm{x}$

13. Thibault R (2010) Assessment of the perioperative nutritional status Nutrition Clinique et metabolisme 24 157-66 DOI: $10.1016 / \mathrm{j}$. nupar.2010.10.005

14. Mullen JT and Moorman DW (2009) The obesity paradox: body mass index and outcome in patients undergoing nonbariatric general surgery Ann Surg 250(1) 166-72 DOI: 10.1097/SLA.0b013e3181ad8935 PMID: 19561456

15. Russo MJ and Hong KN (2010) The effect of body mass index on survival following hearth transplantation: do outcomes support consensus guidelines? Ann Surg 251(1) 144-52 DOI: 10.1097/SLA.0b013e3181b5db3c

16. Aussel C (2013) Is serum albumin a marker of nutritional status? Nutrition Clinique et metabolisme 27 28-33 DOI: $10.1016 / \mathrm{j}$. nupar.2012.12.003

17. Kuzu MA and Terzioglu H (2006) Preoperative nutritional risk assessment in predicting postoperative outcome in patients undergoing major surgery World J Surg 30(3) 378-90 DOI: 10.1007/s00268-005-0163-1 PMID: 16479353

18. Schiesser $M$ and Kirchhoff $P$ (2009) The correlation of nutrition risk index, nutrition risk score, and bioimpedance analysis with postoperative complications in patients undergoing gastrointestinal surgery Surgery 145(5) 519-26 DOI: 10.1016/j. surg.2009.02.001 PMID: 19375611 
19. Appleton J (2002) Arginine: Clinical potential of a semi-essential amino-acid Altern Med Rev 7(6) 512-22 PMID: 12495375

20. Garazi E et al (2013) Inflammation in cancer and immunonutrition: Is the Neutropil-Lymphocyte ratio a promising monitoring? [Abstract] Congrès annuel de la Société des Réanimateurs de Langue Française Paris, France

21. Giger U and Büchler M (2007) Preoperative immunonutrition suppresses perioperative inflammatory response in patients with major abdominal surgery. A randomized controlled pilot study Ann Surg Oncol 14(10) 2798-806 DOI: 10.1245/s10434-0079407-7 PMID: 17632760

22. Roumen RM and Frieling JT (1993) Endotoxemia after major vascular operations J Vasc Surg 18(5) 853-7 DOI: 10.1016/07415214(93)90341-I PMID: 8230573

23. Siddiqui RA and Shaikh SR (2004) Omega 3-fatty acids: health benefits and cellular mechanisms of action Mini Rev Med Chem 4(8) 859-71 DOI: 10.2174/1389557043403431 PMID: 15544547

24. Rainer $\mathrm{H}$ and Böger $\mathrm{J}$ (2007) The pharmacodynamics of L-Arginine Nutr 137(6) 1650-5

25. Dupire S and Wemeau M (2012) Prognostic value of PINI index in patients with multiple myeloma Eur J Haemato/ 88(4) 306-13 DOI: 10.1111/j.1600-0609.2011.01740.x PMID: 22126676

26. Devoto $G$ and Gallo $F(2006)$ Prealbumin serum concentrations as a useful tool in the assessment of malnutrition in hospitalized patients Clinical Chem 52(12) 2281-5 DOI: 10.1373/clinchem.2006.080366

27. Hefti MM, David BA and Chessin B (2009) Severity of inflammation as a predictor of colectomy in patients with chronic ulcerative colitis Dis Colon Rectum 52(2) 193-7 DOI: 10.1007/DCR.0b013e31819ad456 PMID: 19279411 PMCID: 2753491

28. Roopali B Gupta and Noam H (2007) Histologic Inflammation Is a Risk Factor for Progression to Colorectal Neoplasia in Ulcerative Colitis: a cohort Study Gastroenterology 133(4) 1099-105 DOI: 10.1053/j.gastro.2007.08.001 PMID: 17919486 PMCID: $\underline{2175077}$

29. Dittmar T and Zaenker KS (2006) Infection, Inflammation and Neoplasia: An Interdisciplinary Challenge Contrib Microbio/ 13 232-9

30. Albers R, Antoine JM and Bourdet-Sicard R (2005) Markers to measure immunomodulation in human nutrition intervention studies Br J Nutr 94(3) 452-81 DOI: 10.1079/BJN20051469 PMID: 16176618

31. Forget $P$, Dinant $V$ and De Kock $M(2015)$ Is the Neutrophil-to-Lymphocyte Ratio more correlated than C-reactive protein with postoperative complications after major abdominal surgery? PeerJ 13(3) e713 DOI: 10.7717/peeri.713

32. Proctor MJ et al (2011) A comparison of inflammation-based prognostic scores in patients with cancer. A Glasgow Inflammation Outcome Study Eur J Cancer 47(17) 2633-41 DOI: 10.1016/j.ejca.2011.03.028 PMID: 21724383

33. Sorensen D et al (2009) Perioperative immunonutrition in head and neck cancer Laryngoscope 119(7) 1358-64 DOI: $10.1002 /$ lary.20494 PMID: 19459146

34. Davis CJ et al (2012) The use of prealbumin and C-reactive protein for monitoring nutrition support in adult patients receiving enteral nutrition in an urban medical center JPEN J Parenter Enteral Nutr 36(2) 197-204 DOI: 10.1177/0148607111413896 